\title{
To Put Back in the Body or to Drop in the Bucket- Outcome after Using an Extruded Devitalized Segment of Bone in an Open Distal Femoral Fracture
}

\author{
Mohamad Gouse*, Subin Babu, Ravichand Ismavel and Korulla Mani Jacob
}

Department of Orthopaedics, Unit 1, Christian Medical College, Vellore, Tamil Nadu, India

\begin{abstract}
A segmental bone defect caused by an open fracture is a daunting problem to deal with and the traditional treatment options that are currently practiced to treat such conditions include allo or autologous bone graft, distraction osteogenesis and membrane induction. Infrequently the course of treatment is complicated by non-union and infection, thus requiring multiple staged procedures that may affect the functional outcome.

We present a case of a 25 year old gentleman who had sustained an isolated open type III A comminuted distal femur fracture with a part of the diaphyseal distal femur extruding from the body with no soft tissue attached. His plain radiograph showed comminuted distal femur AO C3 fracture. Following debridement, the extruding diaphyseal fragment was washed and cleaned with normal saline and retained inside the body. The limb was stabilized with an external fixator and 48 hours later he was taken for a second debridement wherein a stable internal fixation was done retaining the extruded fragment. Post operatively he recovered well without wound complications or any deep or superficial infection. The follow up radiographs showed that the bone coalesced with an uneventful union and in next 3 years the knee ROM was 0-130 degrees. His lower extremity functional score was (LEFS) 74.

This case is to highlight the importance of judicious insight at times when debridement is the matter of concern, taking into consideration that all reconstructive options are available. The outcome of other staged reconstructive procedures to bridge such bone loss is far more tedious and sure to have a far less efficacious outcome than what has been described here. This report also described about other literature about the option available for such condition.
\end{abstract}

Keywords: Distal femur fracture; Segmental bone loss

\section{Introduction}

A segmental bone defect caused by an open fracture is a daunting problem to deal with and the traditional treatment options that are currently practiced to treat such conditions include allo or autologous bone graft, distraction osteogenesis and membrane induction. Frequently the course of treatment is complicated by non-union and infection, thus requiring multiple staged procedures that may affect the functional outcome. We suggest that surgeons should exercise reasonable judgment at the time of debridement, rather than dropping it in the bucket, loose large bone segments can be considered for retention within the body with meticulous debridement and staged rigid fixation.

\section{Case Report}

We present a case of 25 years old healthy young gentleman, a driver by occupation who had a head on collision with a four wheeler while travelling on a motorised two wheeler and sustained an isolated open type III A comminuted distal femur fracture with a part of the diaphyseal distal femur extruding from the body with extensive striping and no remnant soft tissue attachments (Figure 1). On examination in the emergency room he was conscious, oriented and vitals were stable. There was no history of loss of consciousness. There were two $5^{\star} 5 \mathrm{~cm}$ lacerations present on the anterolateral ascept of distal femur exposing the fracture site. There were no distal neurological or vascular deficits. His plain radiograph showed a comminuted distal femur OA C3 compound fracture (Figure 2). After resuscitation he underwent debridement and the extruding diaphyseal fragment was washed and cleaned with copious normal saline irrigation and retained within the body. The limb was stabilized with an external fixator and 48 hours later he was taken for a second look debridement wherein an internal fixation was performed with an angle stable anatomical distal femur locking plate after retaining the extruded fragment. Post operatively he recovered well without wound infections or any other complication of wound healing. He was kept toe touch weight_bearing for 6 weeks and gradually progressive weight bearing was initiated after 6 weeks.
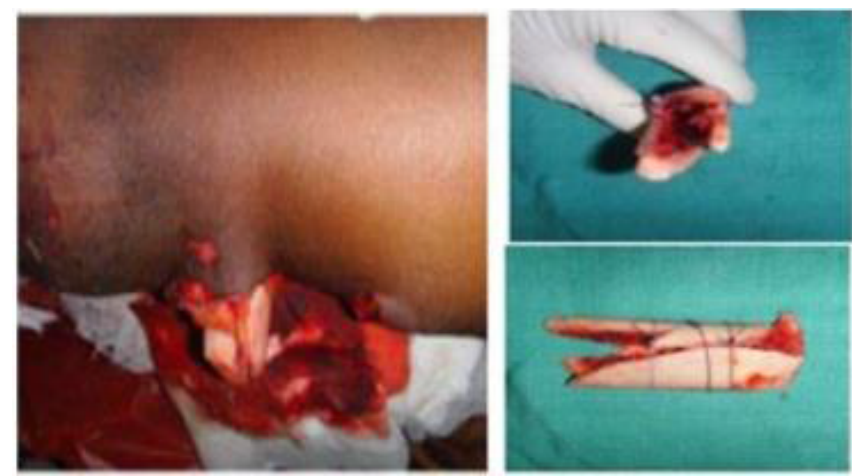

Figure 1: Clinical picture showing open distal femur fracture with extruded diaphyseal fragment.

*Corresponding author: Mohamad Gouse, Department of Orthopaedics, Unit 1, Christian Medical College, Vellore, Tamil Nadu, India; Tel: 0416-2282020; E-mail: gousemohamad@yahoo.com

Received February 23, 2015; Accepted May 26, 2015; Published June 01, 2015

Citation: Gouse M, Babu S, Ismavel R, Jacob KM (2015) To Put Back in the Body or to Drop in the Bucket- Outcome after Using an Extruded Devitalized Segment of Bone in an Open Distal Femoral Fracture. J Trauma Treat 4: 253 doi:10.4172/2167-1222.1000253

Copyright: $\odot 2015$ Gouse M, et al. This is an open-access article distributed under the terms of the Creative Commons Attribution License, which permits unrestricted use, distribution, and reproduction in any medium, provided the original author and source are credited. 
Citation: Gouse M, Babu S, Ismavel R, Jacob KM (2015) To Put Back in the Body or to Drop in the Bucket- Outcome after Using an Extruded Devitalized Segment of Bone in an Open Distal Femoral Fracture. J Trauma Treat 4: 253. doi:10.4172/2167-1222.1000253
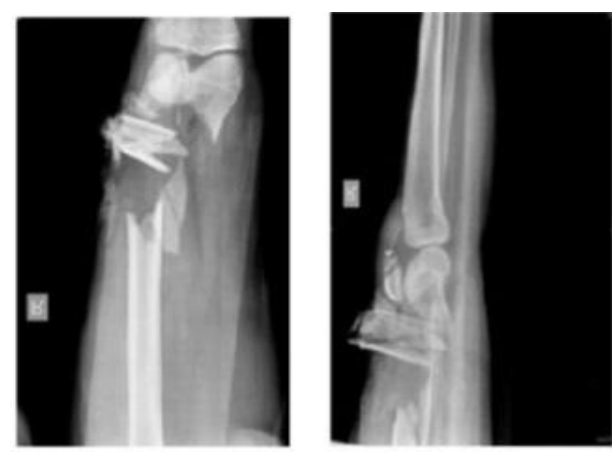

Figure 2: Plain radiograph anterio-posterior and lateral view of distal femur comminuted C3 fracture with segmental bone loss.
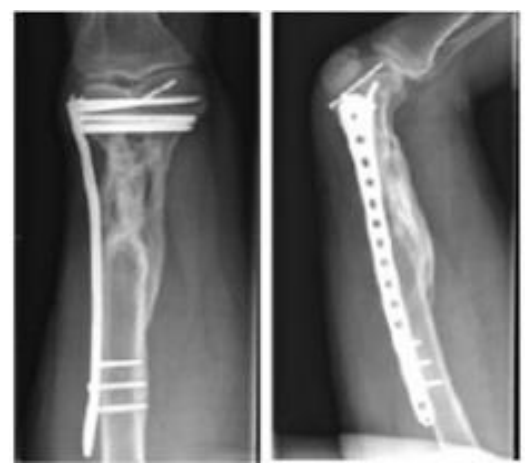

Figure 3: Post-operative plain radiograph AP and LAT after 3 years showing complete union fracture with implants in-situ.
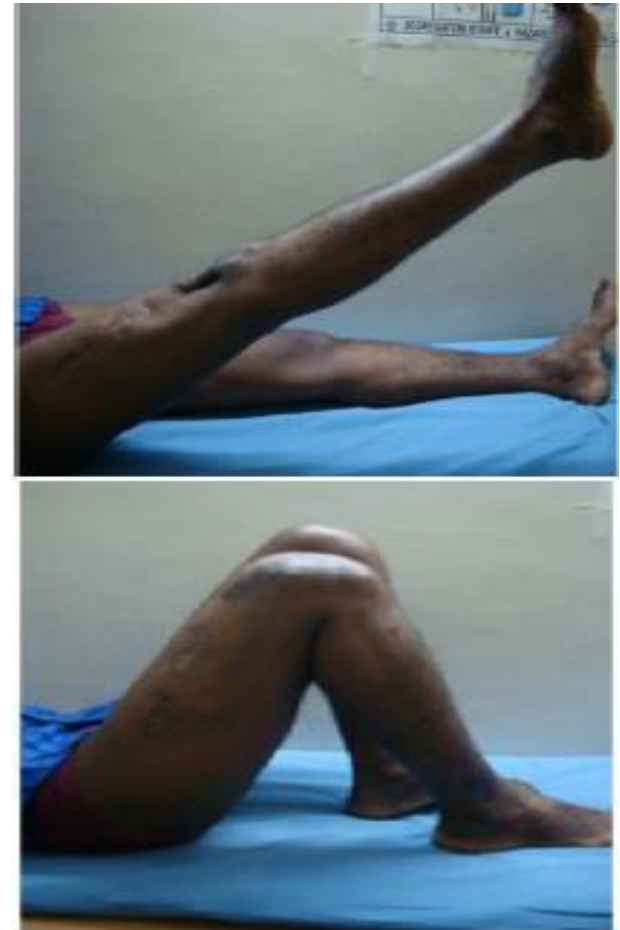

Figure 4: Post-operative plain radiograph AP and LAT after 3 years showing complete union fracture with implants in-situ.
The follow up radiographs showed that the bone coalesced with and incorporated resulting in an uneventful unionunion. In the next 3 years the patient achieved a knee flexion range of 0-130 degrees (Figures 3 and 4). He had a shortening of $2 \mathrm{~cm}$ that was corrected with height correction foot wear and was able to return to his occupation as a professional driver. His lower extremity functional score was (LEFS) 74 at 3 year follow-up.

\section{Discussion}

In spite of recent advancements, the management of complex distal femur fractures is still a challenging problem for orthopedic surgeons due to their guarded outcome. The problem becomes more complicated with segmental bone loss. Presently, there are a very few options that are available to tackle this segmental loss. The Ilizarov technique, vascularized fibular grafts, and acute limb shortening have been used previously to address defects of various lengths; the drawback for these procedures has been pin tract infections and subsequent morbidity $[1,2]$. Traditional bone graft techniques produce unpredictable results and are limited by uncontrollable graft resorption, even when the recipient site is well vascularized [3]. More recently, the use of an antibiotic cement spacer followed by grafting within this space confined by an induced biomembrane has been described as a potential treatment strategy [4,5]. Its drawbacks include donor site morbidity in case of autograft and unavailability, and risk of infection with allograft. In contrast to these, retaining patient's native bone wherever possible with good preparation is a viable option in these difficult situations. Contrary to the existing wisdom which often prescribes excision of extruded bones, this segment of bone was debrided with care, cleaned and incorporated, filling the defect at the time of internal fixation. Though the above is not recommended in literature it may be replicated in selected cases with early presentation, wherein meticulous debridement of the extruded segmented could be retained for the reconstruction.

This case is to highlight the importance of judicious insight at times when debridement is the matter concern, taking into consideration that all reconstructive options are available. The outcome of other staged reconstructive procedures to bridge such bone loss are far more tedious and are sure to have a far less efficacious outcome than what has been described here.

\section{References}

1. Krishnan A, Pamecha C, Patwa JJ (2006) Modified llizarov technique for infected nonunion of the femur: the principle of distraction-compression osteogenesis. J Orthop Surg 14: 265-272.

2. Arazi M, Memik R, Ogün TC, Yel M (2001) Ilizarov external fixation for severely comminuted supracondylar and intercondylar fractures of the distal femur. $J$ Bone Joint Surg Br 83: 663-667.

3. Hertel R, Gerber A, Schlegel U, Cordey J, Rüegsegger P, et al. (1994) Cancellous bone graft for skeletal reconstruction. Muscular versus periosteal bed--preliminary report. Injury 25: A59-70.

4. Masquelet AC, Begue $T$ (2010) The concept of induced membrane for reconstruction of long bone defects. Orthop Clin North Am 41: 27-37.

5. Masquelet AC (2003) Muscle reconstruction in reconstructive surgery: soft tissue repair and long bone reconstruction. Langenbecks Arch Surg 388: 344-346. 